He Yaling, Zeng Shilin, Zhang Peiling* and Xu Zhouqing

\title{
Crystal structure of diaqua-dinitrato- $\mathrm{K}^{2} O, \mathrm{O}^{\prime}((Z)-N-((E)-1-($ pyrazin-2- $y$ l)ethylidene)nicotinohydrazonato- $\mathrm{K}^{3} N, N^{\prime}, O$ )europium(II), $\mathrm{C}_{12} \mathrm{H}_{14} \mathrm{~N}_{7} \mathrm{O}_{9} \mathrm{Eu}$
}

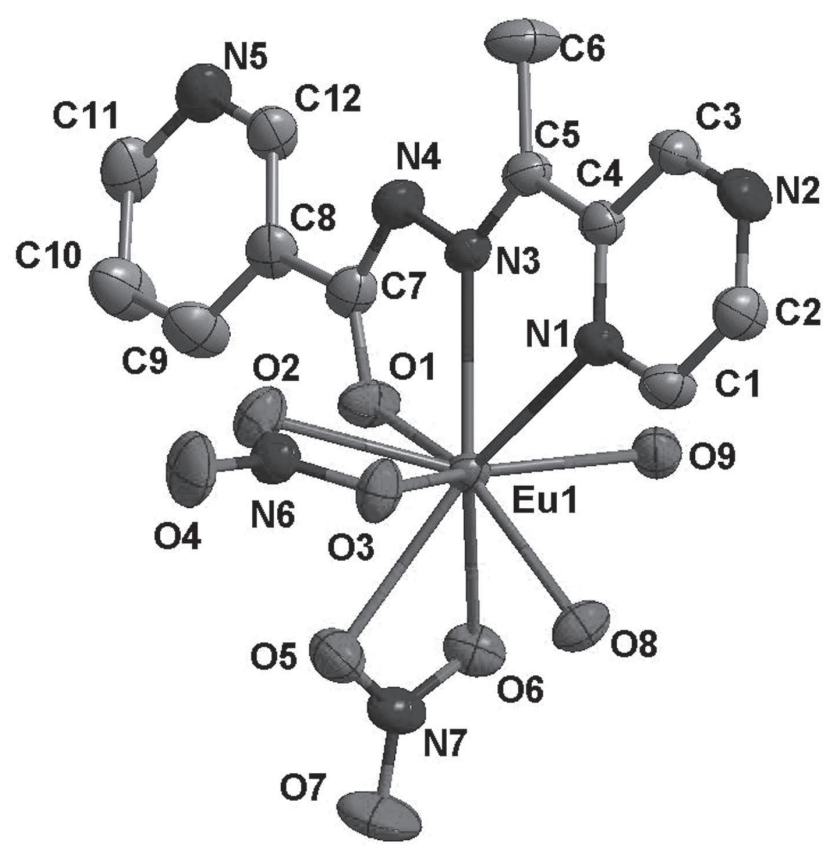

https://doi.org/10.1515/ncrs-2017-0433

Received December 29, 2017; accepted April 12, 2018; available online May 7, 2018

\section{Abstract \\ $\mathrm{C}_{12} \mathrm{H}_{14} \mathrm{~N}_{7} \mathrm{O}_{9} \mathrm{Eu}$, monoclinic, $P 2_{1} / c$ (no. 14), $a=16.770$ (3) $\AA$, $b=7.7185(16) \AA, \quad c=15.165(3) \AA, \quad \beta=108.409(3)^{\circ}$, $V=1862.5(7) \AA^{3}, Z=4, R_{\mathrm{gt}}(F)=0.0174, w R_{\mathrm{ref}}\left(F^{2}\right)=0.0453$, $T=296(2) \mathrm{K}$.}

\section{CCDC no.: 1836606}

The crystal structure is shown in the figure. Hydrogen atoms are omitted for clarity. Tables 1 and 2 contain details on crystal structure and measurement conditions and a list of the atoms including atomic coordinates and displacement parameters.

\section{Source of material}

All chemicals were commercially purchased except for $(E)$ $N^{\prime}$-(1-(pyrazin-2-yl)ethylidene)nicotinohydrazide (HL) which

\footnotetext{
*Corresponding author: Zhang Peiling, College of Physics and Information Engineering, Henan Polytechnic University, Jiaozuo 454000, P.R. China, e-mail: plzhang@hpu.edu.cn

He Yaling, Zeng Shilin and Xu Zhouqing: College of Chemistry and Chemical Engineering, Henan Polytechnic University, Jiaozuo 454000, P.R. China
}

Table 1: Data collection and handling.

\begin{tabular}{ll}
\hline Crystal: & Yellow block \\
Size: & $0.3 \times 0.2 \times 0.2 \mathrm{~mm}$ \\
Wavelength: & Mo $K \alpha$ radiation $(0.71073 \AA)$ \\
$\mu:$ & $3.43 \mathrm{~mm}^{-1}$ \\
Diffractometer, scan mode: & Bruker SMART APEX, $\omega$-scans \\
$\theta_{\max }$, completeness: & $25^{\circ},>99 \%$ \\
$N(h k l)_{\text {measured }}, N(h k l)_{\text {unique }}, R_{\text {int }}:$ & $9021,3265,0.019$ \\
Criterion for $I_{\text {obs }}, N\left(h k l_{\text {gt }}:\right.$ & $I_{\text {obs }}>2 \sigma\left(I_{\text {obs }}\right), 3023$ \\
$N(\text { param })_{\text {refined }}:$ & 265 \\
Programs: & Bruker programs [1], SHELX [2] \\
\hline
\end{tabular}

Table 2: Fractional atomic coordinates and isotropic or equivalent isotropic displacement parameters $\left(\AA^{2}\right)$.

\begin{tabular}{lrrrr}
\hline Atom & $\boldsymbol{x}$ & $\boldsymbol{y}$ & $\boldsymbol{z}$ & $\boldsymbol{U}_{\text {iso }} \boldsymbol{U}_{\text {eq }}$ \\
\hline Eu1 & $0.707812(7)$ & $0.561905(16)$ & $0.307457(8)$ & $0.02413(6)$ \\
C1 & $0.56651(17)$ & $0.3739(4)$ & $0.41599(19)$ & $0.0365(6)$ \\
H1 & 0.5307 & 0.3713 & 0.3550 & $0.044^{*}$ \\
C2 & $0.53898(18)$ & $0.3045(4)$ & $0.4854(2)$ & $0.0407(7)$ \\
H2 & 0.4853 & 0.2573 & 0.4699 & $0.049^{*}$ \\
C3 & $0.66294(19)$ & $0.3749(4)$ & $0.59151(19)$ & $0.0372(6)$ \\
H3 & 0.6981 & 0.3774 & 0.6527 & $0.045^{*}$ \\
C4 & $0.69197(18)$ & $0.4456(3)$ & $0.52348(19)$ & $0.0295(6)$ \\
C5 & $0.77634(18)$ & $0.5215(4)$ & $0.54458(19)$ & $0.0318(6)$ \\
C6 & $0.8338(2)$ & $0.5324(5)$ & $0.6427(2)$ & $0.0533(9)$ \\
H6A & 0.8869 & 0.5799 & 0.6432 & $0.080^{*}$ \\
H6B & 0.8423 & 0.4187 & 0.6696 & $0.080^{*}$ \\
H6C & 0.8091 & 0.6058 & 0.6781 & $0.080^{*}$ \\
C7 & $0.89398(16)$ & $0.6782(4)$ & $0.41603(19)$ & $0.0335(6)$ \\
C8 & $0.97780(17)$ & $0.7587(4)$ & $0.4292(2)$ & $0.0353(6)$ \\
C9 & $0.99715(19)$ & $0.8365(5)$ & $0.3566(2)$ & $0.0504(8)$ \\
H9 & 0.9577 & 0.8384 & 0.2976 & $0.060^{*}$ \\
C10 & $1.0751(2)$ & $0.9117(5)$ & $0.3717(3)$ & $0.0562(9)$ \\
H10 & 1.0884 & 0.9669 & 0.3236 & $0.067^{*}$ \\
C11 & $1.13279(19)$ & $0.9031(4)$ & $0.4595(2)$ & $0.0469(8)$ \\
H11 & 1.1853 & 0.9537 & 0.4699 & $0.056^{*}$ \\
C12 & $1.04039(17)$ & $0.7557(4)$ & $0.5149(2)$ & $0.0386(7)$ \\
H12 & 1.0286 & 0.7021 & 0.5643 & $0.046^{*}$ \\
N1 & $0.64234(14)$ & $0.4442(3)$ & $0.43337(15)$ & $0.0299(5)$ \\
N2 & $0.58747(15)$ & $0.3036(3)$ & $0.57386(16)$ & $0.0410(6)$ \\
N3 & $0.79739(14)$ & $0.5740(3)$ & $0.47362(15)$ & $0.0296(5)$ \\
N4 & $0.87724(14)$ & $0.6442(3)$ & $0.49366(16)$ & $0.0365(5)$ \\
N5 & $1.11609(14)$ & $0.8249(3)$ & $0.53036(18)$ & $0.0426(6)$ \\
N6 & $0.63022(15)$ & $0.8962(3)$ & $0.32196(16)$ & $0.0350(5)$ \\
N7 & $0.68730(16)$ & $0.6104(3)$ & $0.11136(16)$ & $0.0393(6)$ \\
01 & $0.84578(11)$ & $0.6475(3)$ & $0.33349(13)$ & $0.0390(5)$ \\
O2 & $0.70859(12)$ & $0.8844(3)$ & $0.34105(15)$ & $0.0415(5)$ \\
O3 & $0.58882(12)$ & $0.7567(2)$ & $0.30913(14)$ & $0.0385(5)$ \\
O4 & $0.59448(16)$ & $1.0371(3)$ & $0.31490(19)$ & $0.0560(6)$
\end{tabular}


Table 2 (continued)

\begin{tabular}{lrrrr}
\hline Atom & $\boldsymbol{x}$ & $\boldsymbol{y}$ & $\boldsymbol{z}$ & $\boldsymbol{U}_{\text {iso }}{ }^{*} \boldsymbol{U}_{\text {eq }}$ \\
\hline O5 & $0.66480(14)$ & $0.7272(3)$ & $0.15723(14)$ & $0.0454(5)$ \\
O6 & $0.73079(13)$ & $0.4877(3)$ & $0.15779(14)$ & $0.0401(5)$ \\
O7 & $0.66750(19)$ & $0.6168(4)$ & $0.02697(16)$ & $0.0711(8)$ \\
O8 & $0.57973(12)$ & $0.4252(2)$ & $0.21120(14)$ & $0.0359(5)$ \\
H8A & 0.5410 & 0.4402 & 0.2355 & $0.054^{*}$ \\
H8B & 0.5884 & 0.3174 & 0.2074 & $0.054^{*}$ \\
O9 & $0.75003(12)$ & $0.2650(2)$ & $0.32711(13)$ & $0.0372(4)$ \\
H9A & 0.7103 & 0.2038 & 0.3342 & $0.056^{*}$ \\
H9B & 0.7931 & 0.2555 & 0.3749 & $0.056^{*}$ \\
\hline
\end{tabular}

was synthesized according to the literature [3]. A mixture of $\mathrm{HL}(0.05 \mathrm{mmol}, 12.1 \mathrm{mg})$ and $\mathrm{Eu}\left(\mathrm{NO}_{3}\right)_{3} \cdot 6 \mathrm{H}_{2} \mathrm{O}(0.10 \mathrm{mmol}$, $44.6 \mathrm{mg}$ ) disolved in ethanol $(3 \mathrm{~mL})$ and $\mathrm{H}_{2} \mathrm{O}(3 \mathrm{~mL})$ was placed in a glass bottle $(10 \mathrm{~mL})$ at room temperature. Pale yellow block crystals suitable for single-crystal X-ray diffraction were obtained after 3 days.

\section{Experimental details}

The structure was solved by Direct methods and refined with the SHELX crystallographic software package [2]. The hydrogen atoms were placed at calculated positions and refined as riding atoms with isotropic displacement parameters.

\section{Discussion}

Hydrazones are a class of compounds which are well known for their antimicrobial activity [4, 5]. But as hydrazones are prone to undergo degradation and bacteria can develop resistance to them, complexation of hydrazones with biocompatible metal ions is useful in this regard for long-term effectiveness [6]. In the course of our studies on the chemistry of lanthanide compounds, we have prepared and characterized a number of chelates containing hydrazones as ligands. Herein we report a new $\mathrm{Eu}(\mathrm{III})$ complexes derived from
(E)- $N^{\prime}$-(1-(pyrazin-2-yl)ethylidene)nicotinohydrazide (HL), producing complexes of the type $\left[\mathrm{Eu}(L)\left(\mathrm{NO}_{3}\right)_{2}\left(\mathrm{H}_{2} \mathrm{O}\right)_{2}\right]$.

As reported, the title comlex is a mononuclear coordinational compound in which the nine coordinated $\mathrm{Eu}^{3+}$ is surrounded by one hydrozonate, two nitrato ligands and two water molecules. The bond lengths of $\mathrm{Eu}-\mathrm{O}$ and $\mathrm{Eu}-\mathrm{N}$ (2.317(19)-2.646(2) $\AA$ ) in the title complex are comparable with those found in our previous work [7]. In the solid state, the three dimension supermolecules are constructed by intermolecular $\mathrm{O}-\mathrm{H} \cdots \mathrm{O}$ and $\mathrm{O}-\mathrm{H} \cdots \mathrm{N}$ hydrogen bonds between the coordinated water molecules and the $\mathrm{N}$ atoms of ligand and the $\mathrm{O}$ atoms of the nitrate anions.

\section{References}

1. Bruker. SMART and SAINT. Bruker AXS Inc., Madison, WI, USA (2007).

2. Sheldrick, G. M.: Crystal refinement with SHELX. Acta Crystallogr. C71 (2015) 3-8.

3. Das, L. K.; Diaz, C.; Ghosh, A.: Antiferromagnetic mixed-valence $\mathrm{Cu}$ two-dimensional coordination polymers constructed by double oximato bridged Cu dimers and CuISCN based onedimensional anionic chains. Cryst. Growth Des. 15 (2017) 3939-3949.

4. Peng, H.-D.; Yang, J.-H.; Yang, G.-C.; Chen, Z.-X.: Synthesis of 2-thioquinazolin-4-ones. Journal of Hubei University (Natrual Science) 28 (2006) 282-284, 292.

5. Yan, L.-L.; Cai, H.-X.: Crystal structure of 3-benzyl-2,3-dihydro2-thioxoquinazolin-4(1H)-one. Z. Kristallogr. NCS 232 (2017) 811-812.

6. Bakale, R. P.; Pathan, A. H.; Naik, G. N.; Machakanur, S. S.; Mangannavar, C. V.; Muchchandi, I. S.; Gudasi, K. B.: Appl. Organometal. Chem. 28 (2014) 720-724.

7. Xu, Z.-Q.; Mao, X.-J.; Jia, L.; Xu, J.; Zhu, T.-F.; Cai, H.-X.; Bie, H.-Y.; Chen, R.-H.; Ma, T.-L.: Synthesis, characterization and anticancer activities of two lanthanide(III) complexes with a nicotinohydrazone ligand. J. Mol. Struct. 1102 (2015) 86-90. 